\title{
On Centrally Prime and Centrally Semiprime Rings
}

Adil. K. Jabbar

Mathematics Department

College of Science

University of Sulaimani

Received on: 14/08/2006
Abdularahman. H. Majeed

Mathematics Department

College of Science

University of Baghdad

Accepted on: 24/12/2006

ABSTRACT

In this paper, centrally prime and centrally semiprime rings are defined and the relations between these two rings and prime (resp. semiprime) rings are studied.Among the results of the paper some conditions are given under which prime (resp. semiprime) rings become centrally prime (resp.centrally semiprime) as in:1-A nonzero prime (resp. semiprime) ring which has no proper zero divisors is centrally prime (resp.centrally semiprime).Also we gave some other conditions which make prime (resp. semiprime) rings and centrally prime (resp.centrally semiprime) rings equivalent, as in :2-A ring which satisfies the- $(B Z P)$ for multiplicative systems is prime (resp. semiprime) if and only if it is centrally prime (resp.centrally semiprime).3-A ring with identity in which every nonzero element of its center is a unit is prime (resp. semiprime) if and only if it is centrally prime (resp.centrally semiprime).

Keywords: prime rings, semiprime rings, centrally prime rings, centrally semiprime rings, localization.

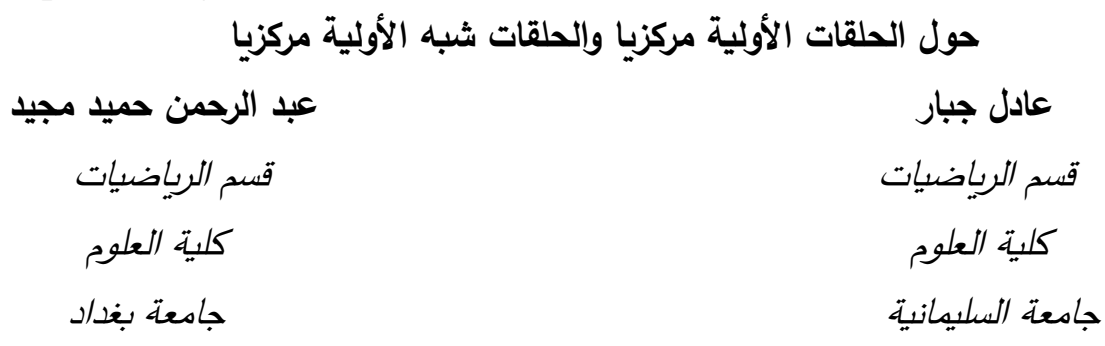

تاريخ القبول: 2006/12/24

تاريخ الاستلام: 2006/8/14

الملخص

في هذا البحث قدمت تعريفالحلقا ت الاولية مركزيا والحلقا ت شبه الاولية مركزيـا وتمت

دراسة العلاقات بين هاتين الحلقتين والحلقات الاولية وشبه الاولية. من بين النتائج التى توصلنا اليها

هى تحديد بعض الشروط التى عند توافرها تصبح الحلقات الاولية (شبه الاولية) حلقات اولية (شبه

اولية) مركزيا كما فى: 1 -الحلقات الاولية (شبه الاولية)غير الصفرية التى ليست لها قواسم صفرية الهرهية

فعلية تكون حلقات اولية(شبه اولية)مركزيا. 
كذلك اعطينا شروطا اخرى تجعل من الحلقات الاولية(شبه الاولية) والحلقات الاولية(شبه الاولية)مركزيا حلقات متكافئة كما فى:2 -الحلقات التى تحقق ( خاصية -ذات الصفرين - ) للانظمة الضربية تكون حلقات اولية(شبه اولية) اذا وفقط اذا كانت حلقات اولية(شبه اولية) مركزيا. 3-الحلقة ذات العنصر المحايدالضربى التى تثكل كل عنصر غير صفرى من عناصر مركزها وحدة تكون اولية(شبه اولية) اذاوفقط اذا كانت اولية(شبه اولية) مركزيا.

الكلمـات المفتاحية: الحلقات الأولية، الحلقات شبه الاولية، الحلقات الأولية مركزيها، الحلقات شبه الديه

Introduction:

$$
\text { الأولية مركزيا. }
$$

Let $R$ be a ring. A non-empty subset $S$ of $R$ is said to be a multiplicative closed set in $R$ if $a, b \in S$ implies that $a b \in S$,(Larsen and McCarthy,1971) and a multiplicative closed set $S$ is called a multiplicative system if $0 \notin S$,(Larsen and McCarthy ,1971).Let $S$ be a multiplicative system in $R$ such that $[S, R]=\{0\}$, where $[S, R]=\{[s, r]: s \in S, r \in R\}$. Define a relation $(\sim)$ on $R \times S$ as follows :

If $(a, s),(b, t) \in R \times S$ then $(a, s) \sim(b, t)$ if and only if there exists $x \in S$ such that $x(a t-b s)=0$. Since $[S, R]=\{0\}$, it can be shown that $(\sim)$ is an equivalence relation on $R \times S$. Now denote the equivalence class of $(a, s)$ in $R \times S$ by $a_{S}$, that is $a_{S}=\{(b, t) \in R \times S:(a, s) \sim(b, t)\}$ (this equivalence class is also denoted by $\frac{a}{s}$ (Larsen and McCarthy,1971) or by $s^{-1} a$, and then denote the set of all equivalence classes determined under this equivalence relation by $R_{S}$, that is let $R_{S}=\left\{a_{S}:(a, s) \in R \times S\right\}$. Note that $R_{S}$ is also denoted by $S^{-1} R$ (Larsen and McCarthy, 1971).

On $R_{S}$ we define addition ( + ) and multiplication (.) as follows: $a_{s}+b_{t}=(a t+b s)_{s t}$ and $a_{s} \cdot b_{t}=(a b)_{s t}$, for all $a_{s}, b_{t} \in R_{S}$.

It can be shown that these two operations are well-defined and that $\left(R_{S},+,.\right)$ forms a ring which is known as the localization of $R$ at $S$ (Larsen and McCarthy, 1971).

Let $R$ be a ring.Then $R$ is called a prime ring if whenever $a, b \in R$ are such that $a R b=\{0\}$ then $a=0$ or $b=0$, (Ashraf,2005, Jung and Park, 2006), and it is called a semiprime ring if $a \in R$, is such that $a R a=\{0\}$ then $a=0$,(Vukman, 1999, Argac, Nakajima and Albas, 2004), where $a R b=\{a r b: r \in R\}$.

Before giving the main results of the paper we introduce some definitions . 
Let $R$ be a ring and $S$ a multiplicative system in $R$. We say $S$ has zero commutator if $[S, R]=\{0\}$ and we call $S$ a bi-zero multiplicative system if:

i: $[S, R]=\{0\}$ and ii: $\operatorname{ann}(s)=\{0\}$, for all $s \in S$, and we say $R$ satisfies the bizero property $(B Z P)$ for multiplicative systems in $R$ if every multiplicative system $S$ in $R$ which has the property $[S, R]=\{0\}$ has also the property that $\operatorname{ann}(s)=\{0\}$, for all $s \in S$.

\section{Example 1:}

It is easy to show that every multiplicative system in $Z$ is a bi-zero multiplicative system, that is if $S$ is any multiplicative system in $Z$ then $[S, R]=\{0\}$ and $\operatorname{ann}(s)=\{0\}$, for all $s \in S$.

Now let us take the ring of all $2 \times 2$ matrices over $Z,\left(M_{2 \times 2},+,.\right)$.It is known that this ring is not commutative. Take $S=\left\{\left(\begin{array}{ll}1 & 0 \\ 0 & 1\end{array}\right),\left(\begin{array}{cc}-1 & 0 \\ 0 & -1\end{array}\right)\right\}$, it is not difficult to show that $S$ is a multiplicative system in $M_{2 \times 2}$, also we can show that $\left[S, M_{2 \times 2}\right]=\{0\}$.

To show ann $\left(\left(\begin{array}{ll}1 & 0 \\ 0 & 1\end{array}\right)\right)=\left\{\left(\begin{array}{ll}0 & 0 \\ 0 & 0\end{array}\right)\right\}=\operatorname{ann}\left(\left(\begin{array}{cc}-1 & 0 \\ 0 & -1\end{array}\right)\right.$.

If $\left(\begin{array}{ll}x & y \\ u & v\end{array}\right) \in \operatorname{ann}\left(\left(\begin{array}{ll}1 & 0 \\ 0 & 1\end{array}\right)\right)$, then $\left(\begin{array}{ll}1 & 0 \\ 0 & 1\end{array}\right) \cdot\left(\begin{array}{ll}x & y \\ u & v\end{array}\right)=\left(\begin{array}{ll}0 & 0 \\ 0 & 0\end{array}\right)$ which implies that $\left(\begin{array}{ll}x & y \\ u & v\end{array}\right)=\left(\begin{array}{ll}0 & 0 \\ 0 & 0\end{array}\right)$, and hence $\operatorname{ann}\left(\left(\begin{array}{ll}1 & 0 \\ 0 & 1\end{array}\right)\right)=\left\{\left(\begin{array}{ll}0 & 0 \\ 0 & 0\end{array}\right)\right\}$.

Similarly it can be shown that $\left.\operatorname{ann}\left(\begin{array}{cc}-1 & 0 \\ 0 & -1\end{array}\right)\right)=\left\{\left(\begin{array}{ll}0 & 0 \\ 0 & 0\end{array}\right)\right\}$. That means it is also possible for non commutative rings to have multiplicative systems with above two properties.

\section{Remark:}

Let $R$ be a ring and $S$ a multiplicative system in $R$ such that $[S, R]=\{0\}$.If $A$ is an ideal of $R$ then it is easy to show that $A_{S}=\left\{a_{S}: a \in A, s \in S\right\}$ is an ideal of $R_{S}$.

Conversly, if $K$ is an ideal in $R_{S}$ then there exists an ideal $J$ in $R$ such that $K=J_{S}$,(Jabbar,2004).It is necessary to mention that if $A, B$ are ideals of $R$ such that $A=B$ then $A_{S}=B_{S}$ but in general the converse is not true and we give below an example to establish this fact.

\section{Example 2:}


Consider the ring $\left(Z_{12},{ }_{12},{ }_{12}\right) . S=\{1,2,4,8\}$ is a multiplicative system in $Z_{12}$.By simple computation we can get:

$\left(Z_{12}\right)_{S}=\left\{0_{1}, 0_{2}, 0_{4}, 0_{8}, 1_{1}, 1_{2}, 1_{4}, 1_{8}, 2_{1}, 2{ }_{2}, 2_{4}, 2{ }_{8}, \ldots, 11_{1}, 11_{2}, 11_{4}, 11_{8}\right\}$.

Note that

$0_{1}=0_{2}=0_{4}=0_{8}=3_{1}=3_{2}=3_{4}=3_{8}=6_{1}=6_{2}=6_{4}=6_{8}=9_{1}=9_{2}=9_{4}=9_{8}$,

$1_{1}=1_{4}=2_{2}=2_{8}=4_{1}=4_{4}=5_{2}=5_{8}=7_{1}=7_{4}=8_{2}=8_{8}=10_{1}=10_{4}=11_{2}=11_{8}$ and

$2_{1}=1_{2}=1_{8}=2_{4}=4_{2}=4_{8}=5_{1}=5_{4}=7_{2}=7_{8}=8_{1}=8_{4}=10_{2}=10_{8}=11_{1}=11_{4}$.

So that $\left(Z_{12}\right)_{S}=\left\{0_{1}, 1_{1}, 2_{1}\right\}$ which is an ideal in $\left(Z_{12}\right)_{S}$.

Now $I=\{0,2,4,6,8,10\}$ and $J=\{0,4,8\}$ are two ideals in $Z_{12}$ so that $I_{S}, J_{S}$ are ideals in

$\left(Z_{12}\right)_{S}$. By the same technique as we used above we can get that $I_{S}=\left\{0_{1}, 1_{1}, 2_{1}\right\}$ and $J_{S}=\left\{0_{1}, 1_{1}, 2_{1}\right\}$. So that $I_{S}=\left(Z_{12}\right)_{S}=J_{S}$ but $I \neq J$.

and also we will show that this existence becomes unique under certain conditions as we see latter(see Theorem 2 and Theorem 3). Now we mention the following two results the proof of which could be found in (Jabbar,2004).

Let $R$ be a ring and $S$ is a multiplicative system in $R$ such that $[S, R]=\{0\}$.If $I$ and $J$ are ideals of $R$ then:

1: $(I J)_{S}=I_{S} J_{S}$, and 2: $\left(I^{n}\right)_{S}=\left(I_{S}\right)^{n}$, for all $n \in Z^{+}$.

\section{The Main Results:}

First we prove a lemma which will play the basic role ,as we see latter, in the proof of the main results of the paper.

\section{Lemma 1:}

Let $R$ be a ring and $S$ a bi-zero multiplicative system in $R$.If $a, b \in R$ and $s, t \in S$ then $a R b=\{0\}$ if and only if $a_{S} R_{S} b_{t}=\{0\}$.

\section{Proof :}

Now let $a R b=\{0\}$.Then if $r_{x} \in R_{S}$ (is any element), where $r \in R$ and $x \in S$, we have $a_{s} r_{x} b_{t}=(a r b)_{s x t}=0_{s x t}=0$, (since $a r b \in a R b=\{0\}$, so $\left.a r b=0\right)$.

Hence $a_{s} r_{x} b_{t}=0$, for all $r_{x} \in R_{S}$, thus $a_{S} R_{S} b_{t}=\{0\}$, which proves the "only if " part.

To prove the "if"part, let $a_{S} R_{S} b_{t}=\{0\}$, where $a, b \in R$ and $s, t \in S$, then for any $r \in R$ we have $r_{s} \in R_{S}$, and hence $a_{s} r_{s} b_{t} \in a_{S} R_{S} b_{t}$, which gives $a_{s} r_{s} b_{t}=0$ or $(a r b)_{s s t}=0$, then there exists $t \in S(t$ depends on $r)$ such that $t(\operatorname{arb})=0$, thus $\operatorname{arb} \in \operatorname{ann}(t)$ and $S$ bieng a bi-zero multiplicative system so 
$\operatorname{ann}(t)=0$ and hence we get $\operatorname{arb}=0$, this last result is true for all $r \in R$, which implies that $a R b=\{0\}$, and this completes the proof

\section{Remark:}

If $R$ is a ring and $S$ is a multiplicative system in $R$ such that $[S, R]=\{0\}$.If $I, J$ are ideals in $R$ such that $I=J$ then $I_{S}=J_{S}$. But in general the converse is not true (see Example 2). Now we give some conditions under which $I_{S}=J_{S}$ implies $I=J$.

\section{Theorem 2:}

Let $R$ be a ring and suppose that $S$ is a multiplicative system in $R$ such that $[S, R]=\{0\}$.

If $I$ and $J$ are prime ideals of $R$ such that $I \cap S=\phi=J \cap S$, then $I=J$ if and only if $I_{S}=J_{S}$.

\section{Proof:}

For the proof of the "only if" part see the last remark so we prove only the "if"part.

Let $I_{S}=J_{S}$. To show $I=J$. Let $a \in I$. Since $S \neq \phi$ so take $s \in S$.Then $a_{S} \in I_{S}$ andhence $a_{s} \in J_{S}$, and so $a_{S}=b_{t}$ for some $b \in J, t \in S$, so that $(a, s) \sim(b, t)$ which implies that there exists $u \in S$ such that $u(a t-b s)=0$, then $u a t=u b s \in J$ or $u t a \in J$ but $u, t \in S$ implies $u t \in S$ and $J \cap S=\phi$ thus $u t \notin J$ and $J$ being a prime ideal so $a \in J$.Hence $I \subseteq J$.By the same technique we can show that $J \subseteq I$ and hence $I=J$

\section{Theorem 3:}

Let $R$ be a ring with identity 1, s is a multiplicative system in $R$ such that $[S, R]=\{0\}$ and $I, J$ are ideals in $R$.If every non-zero element of Cent $R$ is a unit in $R$, then $I=J$ if and only if $I_{S}=J_{S}$.

\section{Proof:}

The "only if " part has been proved.So it remains to prove the converse part.

Let $I_{S}=J_{S}$.If $x \in I$ then there exists $s \in S$, (since $\left.S \neq \phi\right)$ and then $x_{s} \in I_{S}=J_{S}$, and hence there exists

$a_{t} \in J_{S}$, for some $a \in J$ and $t \in S$ such that $x_{S}=a_{t}$ which gives $(x, s) \sim(a, t)$, hence there exists $v \in S$ such that $v(x t-a s)=0$ or $v x t=v a s \in J$ then $v t x \in J$. Now $v, t \in S$ implies $v t \in S$, thus $v t \neq 0$ (since $0 \notin S$ ). But then since $[S, R]=\{0\}$ so $[v t, R]=\{0\}$ which means that $v t \in C e n t R$, hence $0 \neq v t \in C e n t R$ and thus by the given assumption $v t$ is a unit in $R$, that is $(v t)^{-1} \in R$ and then $v t x \in J$ 
implies $(v t)^{-1} v t x \in J$ that is $1 . x \in J$ which means that $x \in J$ and hence

$I \subseteq J$.Similarly it can be shown that $J \subseteq I$.Hence $I=J \bullet$

Now we introduce the following definition:

\section{Definition:}

Let $R$ be a ring.We say that $R$ is centrally prime (resp. centrally semiprime) if $R_{S}$ is prime (resp. semiprime) for all multiplicative systems $S$ in $R$ which have zero commutators.

\section{Example 3:}

As we have mentioned in Example 1, that every multiplicative system $S$ in $Z$ is a bi-zero multiplicative system, that is $Z$ satisfies the $-(B Z P)$ property.Now we will show that $Z_{S}$ is a prime (resp. semiprime) ring.Now let for $a, b \in Z$ and $s, t \in S$ we have $a_{s} Z_{S} b_{t}=\{0\}$, but $S$ being a bi-zero multiplicative system so by Lemma 1, we get that $a Z b=\{0\}$, and hence as especial case $a .1 . b=0$ or $a . b=0$, which implies $a=0$ or $b=0$, thus $a_{S}=0_{s}=0$ or $b_{t}=0_{t}=0$, so $Z_{S}$ is a prime ring, and $S$ being arbitrary multiplicative system with zero commutator, so we get that $Z$ is a centrally prime ring .Since every prime ring is a semiprime ring so every centrally prime ring is centrally semiprime and thus $Z$ is also a centrally semiprime ring.

Next we apply the result of Lemma 1, to prove some theorems which determine the relations between prime (resp. semiprime) and centrally prime(resp.centrally semiprime) rings, in each of the following two theorems (Theorem 4 and Theorem 5) a condition is given which makes prime (resp. semiprime) rings and centrally prime (resp. centrally semiprime) rings equivalent.

\section{Theorem 4 :}

Let $R$ be a ring. If $R$ satisfies the-(BZP) for multiplicative systems, then $R$ is prime (resp. semiprime) if and only if $R$ is centrally prime (resp. centrally semiprime).

\section{Proof :}

Let $R$ be a prime ring and $S$ be any multiplicative system in $R$ which has zero commutator, that is $[S, R]=\{0\}$, to show $R$ is a centrally prime ring it is enough to show that $R_{S}$ is a prime ring. Since $R$ satisfies the- $(B Z P)$ so $a n n(s)=\{0\}$, for all $s \in S$, that is $S$ is a bi-zero multiplicative system. Now let for $a_{S}, b_{t} \in R_{S}$ we have $a_{S} R_{S} b_{t}=\{0\}$, (where $a, b \in R$ and $s, t \in S$ ).Then by Lemma 1 , we get $a R b=\{0\}$ and $R$ being a prime ring we 
get $a=0$ or $b=0$.If $a=0$ then $a_{S}=0_{s}=0$ and if $b=0$ then $b_{t}=0_{t}=0$ and hence $R_{S}$ is a prime ring and since $S$ is arbitrarily choosen we get that $R_{S}$ is prime for each multiplicative system $S$ in $R$ which has zero commutator and hence $R$ is centrally prime .

Conversely, let $R$ be centrally prime ring, we will show that $R$ is prime. So let for $a, b \in R$ we have $a R b=\{0\}$, if $S$ is any multiplicative system in $R$ with zero commutator then $R_{S}$ is a prime ring and since $R$ satisfies (BZP) for multiplicative systems so $S$ has the property that $\operatorname{ann}(s)=\{0\}$, for all $s \in S$, that is $S$ is a bi-zero multiplicative system. Now since $S \neq \phi$, so there exists an $s \in S$, then $a_{S}, b_{S} \in R_{S}$, and since $a R b=\{0\}$ and $S$ is a bi-zero multiplicative system so by Lemma 1, we get $a_{S} R_{S} b_{S}=\{0\}$, but $R_{S}$ being a prime ring we get $a_{S}=0$, or $b_{S}=0$. If $a_{S}=0$, then there exists $u \in S$ such that $u a=0$, thus $a \in \operatorname{ann}(u)$ and since $R$ satisfies $(B Z P)$ for multiplicative systems so $\operatorname{ann}(u)=\{0\}$ and hence $a=0$.If $b_{S}=0$, by the same technique we get $b=0$. Hence $R$ is a prime ring which completes the proof of the case when $R$ is prime and for the case when $R$ is semiprime the same technique is applicable to get the result $\bullet$

\section{Remark:}

In the Example 3, we have proved directly that $Z$ is a centrally prime as well as a centrally semiprime ring, here we can use Theorem 4, to show this fact as follows:

It is known that a non-zero ring which has no zero divisors are prime as well as semiprime and since $Z$ has no zero divisors so it is prime and hence semiprime, on the other hand $Z$ satisfies the $(B Z P)$ for multiplicative systems as we have mention in Example 3, thus by applying Theorem 4, $Z$ becomes centrally prime and hence centrally semiprime .

\section{Theorem 5:}

Let $R$ be a ring.If $R$ has the identity 1 and every nonzero element of Cent $R$ is a unit in $R$ then $R$ is a prime (resp. semiprime) ring if and only if it is centrally prime (resp. centrally semiprime).

\section{Proof:}

We will show that $R$ satisfies the- (BZP) for multiplicative systems in $R$,so let $S$ be any multiplicative system in $R$ with zero commutator.If $s \in S$ is any element then since $[S, R]=\{0\}$ so $[s, r]=0$, for all $r \in R$, that is $s r-r s=0$, for all $r \in R$, and thus $s r=r s$, for all $r \in R$ which means that $s \in C e n t R$, and then $0 \notin S$ and $s \in S$ implies $s \neq 0$, hence $0 \neq s \in C e n t R$ which means that $s$ is a unit, the next step is to show that $\operatorname{ann}(s)=\{0\}$, so let 
$x \in \operatorname{ann}(s)$ then $s x=0$ and $s$ being a unit,$s^{-1} \in R$ thus $s x=0$ implies that $s^{-1} s x=s^{-1} 0=0$, hence $x=0$ which means $\operatorname{ann}(s)=\{0\}$, and $s$ being arbitrary in $S$ so we get that $\operatorname{ann}(s)=\{0\}$, for all $s \in S$, and hence every multiplicative system $S$ in $R$ with zero commutator satisfies also the property $\operatorname{ann}(s)=\{0\}$, for all $s \in S$, and thus $R$ satisfies (BZP) for multiplicative systems.Hence by Theorem 4, $R$ is prime (resp. semiprime) if and only if it is centrally prime (resp.centrally semiprime) which completes the proof $\bullet$ Now, in each of the following two theorems we give a condition which makes prime (resp. semiprime) rings centrally prime (resp. centrally semiprime), we see below that nonzero prime (resp. semiprime) rings which have no zero divisors are centrally prime (resp. centrally semiprime), which means, in some sense, that centrally prime (resp. centrally semiprime) rings are generalizations of those non-zero rings which have no proper zero divisors.

\section{Theorem 6 :}

If $R$ is a non-zero prime (resp. semiprime) ring which has no proper zero divisors then it is centrally Prime (resp. centrally semiprime).

\section{Proof:}

Suppose $R$ has no proper zero divisors.We will show that $R$ is centrally prime, so let $S$ be any multiplicative system in $R$ with zero commutator, to show that $R_{S}$ is a prime ring, let for $a_{S}, b_{t} \in R_{S}$ we have $a_{S} R_{S} b_{t}=\{0\}$, where $a, b \in R$ and $s, t \in S$. Since $R \neq\{0\}$, so there exists $0 \neq r \in R$. Then $r_{s} \in R_{S}$ and hence $a_{S} r_{s} b_{t} \in a_{S} R_{S} b_{t}$ which gives $a_{s} r_{S} b_{t}=0$ or $(a r b)_{s s t}=0$, and hence we get that there exists $t \in S$ such that $t(a r b)=0$ or tarb $=0$, but $R$ has no proper zero divisors so $t=0$ or $a=0$ or $r=0$ or $b=0$. But $0 \notin S$ and $t \in S$ implies that $t \neq 0$. Also $r \neq 0$ (since $\mathrm{r}$ is choosen non-zero in $R$ ) thus we get $a=0$ or $b=0$. If $a=0$ then $a_{S}=0_{S}=0$ and if $b=0$ then $b_{t}=0_{t}=0$.

Hence $R_{S}$ is a prime ring and $S$ being arbitrary multiplicative system in $R$ with zero commutator we get $R_{S}$ which is a prime ring for all multiplicative systems $S$ in $R$ with zero commutators and hence $R$ is a centrally prime ring. The proof of semiprimeness case is exactly as the proof of primeness case but we just take $a_{S} R_{S} a_{S}=\{0\}$ instead of $a_{S} R_{S} b_{t}=\{0\}$ and repeating the same outlines of the above proof $\bullet$.

Next we give another condition under which prime (resp. semiprime) rings are centrally 
prime (resp. centrally semiprime) and that condition provides $R$ to be a finite ring and this can regarded as a corollary to Theorem 6.

\section{Corollary 7 :}

A finite prime (resp.semiprime) ring $R$ is centrally prime (resp. centrally semiprime).

\section{Proof:}

We will show only the case when $R$ is a prime ring and the case when $R$ is semiprime can be done by the same technique. So let $R=\left\{r_{1}, r_{2}, \ldots, r_{n}\right\}$ and $S$ be any multiplicative system in $R$ with zero commutator, we must show that $R_{S}$ is a prime ring. Now let $a_{S}, b_{t} \in R_{S}$ are such that $a_{S} R_{S} b_{t}=\{0\}$, where $a, b \in R$ and $s, t \in S$.

For each $i(1 \leq i \leq n)$ we have $\left(r_{i}\right)_{S} \in R_{S}$ and thus $a_{S}\left(r_{i}\right)_{S} b_{t}=0$, for all $i$, or $\left(a r_{i} b\right)_{s s t}=0$, for all $i$, and thus we get that for each $i(1 \leq i \leq n)$, there exists $t_{i} \in S$ such that $t_{i}\left(a r_{i} b\right)=0$.Now let $x=t_{1} t_{2} \ldots t_{n}$. Since for all $i, t_{i} \in S$ so that $x=t_{1} t_{2} \ldots t_{n} \in S$. But since $[S, R]=\{0\}$ and for all $i$, we have $t_{i} \in S$, so $t_{i} t_{j}=t_{j} t_{i}$, for all $i, j$.Hence for each $i$ we get $x\left(a r_{i} b\right)=t_{1} t_{2} \ldots t_{n}\left(a r_{i} b\right)=t_{1} t_{2} \ldots t_{i-1} t_{i+1} \ldots t_{n} t_{i}\left(a r_{i} b\right)=t_{1} t_{2} \ldots t_{i-1} t_{i+1} \ldots t_{n} .0=0$, which gives that $(x a) r_{i} b=0$, for all $i$, that is $(x a) r_{i} b=0$, for all $r_{i} \in R$ and this means $(x a) R b=\{0\}$, but $R$ being a prime ring we get $x a=0$ or $b=0$. If $x a=0$ then $a_{s}=x_{x} a_{s}=(x a)_{x s}=0_{x s}=0$, and if $b=0$ then $b_{t}=0_{t}=0$. Hence $R_{S}$ is a prime ring which proves that $R$ is centrally prime $\bullet$. In fact, Corollary 7, tells us that centrally prime (resp.centrally semiprime) rings are, in some sense, generalizations of finite prime (resp. semiprime) rings. 


\section{REFERENCES}

[1] Argac N.,Nakajima A. and Albas E. ;(2004)“ On Orthogonal Generalized Derivations of Semiprime Rings” Turk J Math ,28,185194.

[2] Ashraf M. ;(2005) "On Left $(\vartheta-\phi)$-Derivations of Prime Rings" Archivum Mathematicum (BRNO) Tomus 41,157-166.

[3] Jabbar A.K. ;(2004)"Almost Noetherian Domains which are Almost Dedekind" KAJ, 3(1) Part A , 33-39.

[4] Larsen M.D. and McCarthy P.J. ;(1971) "Multiplicative Theory of Ideals"Academic Press New York and London .

[5] Jung Y.S. and Park K.H. ;(2006)" On generalized $(\alpha-\beta)$ - derivations and commutativity in prime rings" Bull. Korean Math. Soc. 43 , No.1, 101-106.

[6] Vukman J. ;(1999)"An identity related to centralizers in semiprime rings" Comment. Math. Univ. Carolinae 40, No.3, 447-456. 